\title{
AWARE: Platform for Autonomous self-deploying and operation of Wireless sensor-actuator networks cooperating with unmanned AeRial vehiclEs.
}

\author{
Anibal Ollero \\ AICIA-Univ Sevilla \\ Camino Descubrimientos \\ 41092 Sevilla, Spain \\ aollero@cartuja.us.es
}

Pedro J. Marron

University of Stuttgart

Universitaetsstr. 38

D-70569 Stuttgart, Germany

marron@informatik.unistuttgart.de

\author{
Markus Bernard \\ Technische Universität Berlin \\ Einsteinufer 17, EN10 \\ D-10587 Berlin, Germany \\ berni@cs.tu-berlin.de \\ Jason Lepley \\ SELEX SAS Ltd. \\ Christopher Martin Road \\ Basildon, Essex, SS14 3EL, UK \\ Jason.lepley@selex-sas.com
}

\author{
Marco La Civita \\ Flying-Cam \\ Pasage d'Eau, $1^{a}$ \\ 4681Oupeye, Belgium \\ mlacivita@flying-cam.com
}

\section{Eduardo de Andrés \\ Iturri}

Galileo Galilei, 25 (P.I. La Garena)

Alc. Henares 28806 Madrid, Spain edeandres@iturri.com

\begin{abstract}
This paper presents the AWARE platform that seeks to enable the cooperation of autonomous aerial vehicles with ground wireless sensor-actuator networks comprising both static and mobile nodes carried by vehicles or people. Particularly, the paper presents the middleware, the wireless sensor network, the node deployment by means of an autonomous helicopter, and the surveillance and tracking functionalities of the platform. Furthermore, the paper presents the first general experiments of the AWARE project that took place in March 2007 with the assistance of the Seville fire brigades.
\end{abstract}

Keywords: wireless sensors and actuator networks, middleware, embedded systems, co-operative robotics, $U$ AVs.

\section{INTRODUCTION}

The general objective of this paper is to presents the general characteristics of the AWARE platform for autonomous self-deploying and operation of wireless sensor-actuator networks cooperating with unmanned aerial vehicles (UAVs). In recent years significant progress in Unmanned Aerial Vehicles (UAVs) [1] and Wireless Sensor Networks (WSNs) [4] have been experienced. The UAVs are now able to be coordinated for missions such as the detection and monitoring of events [1]. On the other hand, WSNs with static nodes have been developed and applied for detection and monitoring activities [3]. However, static WSNs have important limitations as far as the required coverage and the short communication range in the nodes are concerned. The use of mobile nodes can provide the ability to dynamically adapt the network to environmental events and to improve the network connectivity in case of static nodes failure. Moreover, mobile nodes with single-hop communication and the ability to recharge batteries, or refueling, have been proposed as data mules of the network saving energy in static node communications [5]. However, in many scenarios, the motion of the mobile nodes installed on ground vehicles or carried by persons is very constrained, due to the characteristics of the terrain or the dangerous conditions involved. These scenarios can be found in civil security and disasters, but also in other applications such as cinematography and documentary filming. In these scenarios, the use of UAVs is very suitable. The cooperation of these UAVs with the ground wireless sensor network offers many potential applications. However, flight endurance and range of the currently available low cost unmanned aerial vehicles are very constrained. Moreover reliability and fault-tolerance is a main issue in the cooperation of the aerial vehicles. Furthermore, these autonomous vehicles need communication infrastructure to cooperate or to be tele-operated by humans in emergency conditions. Usually this infrastructure is not available, the required communication range is not enough, or the infrastructure is damaged. Then, the network deployment, and the repairing of the coverage or the connectivity of the network by adding suitable sensor and communication elements are major issues

The general objective of the AWARE project is the design, development and experimentation of a platform providing the middleware and all the functionalities required for the cooperation of UAVs, i.e. autonomous helicopters, with a ground sensor-actuator wireless network, including ground mobile nodes carried by persons and vehicles.

Section II of this paper presents the AWARE platform. Sections III, IV and $\mathrm{V}$ will be devoted respectively to summarize the Middleware, WSN, and the autonomous self-deploying system using autonomous helicopters. Section VI presents some functionalities and Section VII will describe some results of the first general AWARE experiments. Finally, sections VIII and IX are devoted to the Conclusions and 
References.

\section{THE AWARE PLATFORM}

The platform was designed to enable the operation in sites with difficult or impossible access and without communication infrastructure. Then, the project considers the self-deploying of the network by means of autonomous helicopters with the ability to transport and deploy loads (communication equipment and nodes of the ground network).

Two different application scenarios are being considered in the AWARE project: Disaster Management and Civil Security, and Filming. Figure 1 illustrates the first scenario. This paper is devoted to analyze the AWARE system in this scenario. However, it should be noted that the localization and tracking functionalities that are being developed are also very valuable for filming

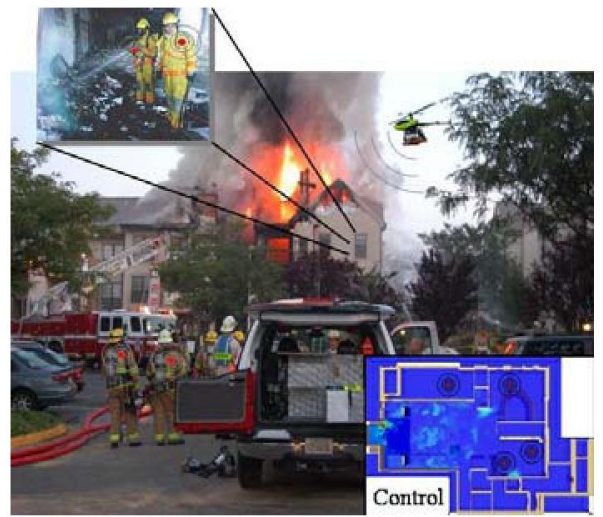

Fig. 1. Disaster Management and Civil Security Applications

The architecture was designed for the cooperation of heterogeneous objects including aerial vehicles, static sensor-actuator nodes, and mobile nodes carried by ground vehicles and persons. The platform consists of two different networks, a High Bandwidth Network (HBN) and a Low Bandwidth Network (LBN), connected through gateways. $\mathrm{HBN}$ is composed of personal computers, Ground Camera Nodes (GCN), and mobile robots capable of transmitting data through IEEE 802.3 or IEEE 802.11 networks. The LBN consist of the nodes of the WSN having very limited computing and data transmitting capabilities. Some mobile robots might be part of both networks. This situation is shown in Fig. 2, where a UAV and an autonomous ground vehicle both have WSN nodes attached. Any device capable of direct communication with both networks might act as a gateway.

\section{MIDDLEWARE}

The platform requires an appropriate middleware [6] that facilitates the communications allowing for topology changes and simplifying the application development in particular scenarios.

The main goal and purpose of the middleware designed and implemented for AWARE is to allow for the communication of heterogeneous entities from the HBN and LBN. The middleware uses the well known publish/subscribe interface and manages the data and control flow between arbitrary devices in the system using data channels. In the project, we have developed our own middleware since due to the special characteristics of the system regarding mobility, device heterogeneity, differences in resources, etc. it was not possible to find a standard middleware solution that can work as a COTS (Commercial off-the-shelf) component. For example, standard solutions in the field of distributed systems such as CORBA do not take mobility into account and fail to work in disconnected environments. In the area of sensor networks, middleware approaches such as TinyDB do not provide the right level of abstraction and do not allow optimizations to be done by the application based on context information.

The current version of the middleware contains three main components: Routing Engine, Filtering Engine and Gateway Management Engine.

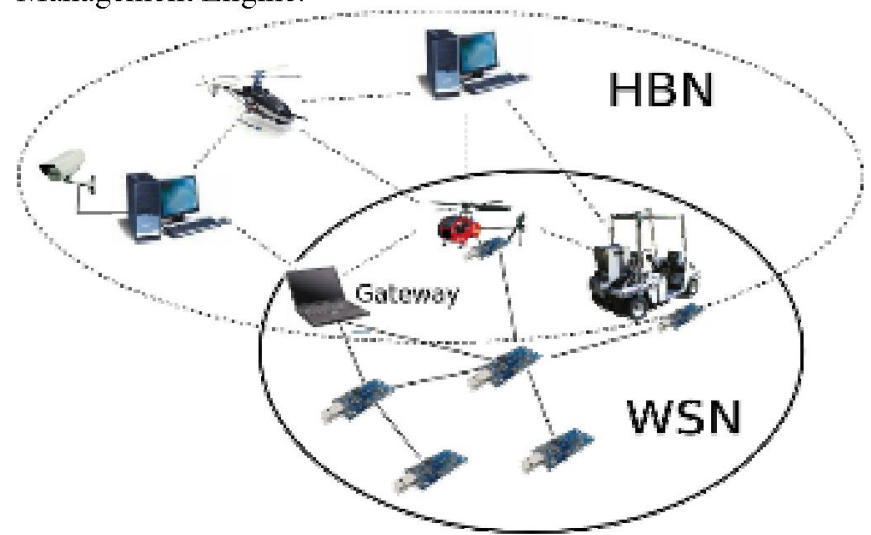

Fig. 2: High Bandwidth and Low Bandwidth Networks

\section{A. Routing Engine}

This component is responsible for the efficient transfer of data from publishers to subscribers, even in the presence of mobility and transient disconnection. The engine implements a pluggable interface for routing algorithms that allow us to replace the routing algorithm as needed. Currently there is support for unreliable and reliable routing based on variants of flooding, gossiping, ODMRP, AODV, as well as geographic routing.

\section{B. Filtering Engine}

This component is responsible for the processing and aggregation of data, message suppression, etc. based on the content provided by the publishers and the current subscriptions registered in the system. Filtering is performed using the following parameters: location information, subscribed data rate and channel content. The filtering engine works very tightly with the routing engine in order to efficiently limit the number of messages sent through the 
network using the routing information found in the system. The novelty of the system is based on the tight coupling of the routing algorithm with the filtering engine that allows the middleware to perform packet level optimizations based on content and not only on addressing. Additionally, location information is used as an intrinsic part of the publish/subscribe system. Location is one of the main factors used in the filtering engine to reduce the number of packets sent within the system and implement the notion of locality required by the scalability of mobile ad-hoc systems.

\section{Gateway Management Engine}

Gateways are the connecting devices between the HBN and the LBN. Since sensors have a limited operational range, it is possible to have a deployment where several physical gateways connect independent sensor networks with the rest of the system. However, our goal is to have a logical view of the sensor nodes. Therefore, the Gateway Management Engine abstracts the usage of several physical gateways by providing one logical gateway to the rest of devices. The optimal selection of the physical gateway as well as the distribution of control information among them is handled transparently by this component.

\section{WIRELESS SENSOR NETWOK}

\section{A. Objectives and challenges}

The objective of the AWARE ground sensor network (GSN) is to provide a description of the physical environment of the deployment area. By means of sensors and wireless communication, the GSN is able to detect events and to collaborate with other entities in the AWARE platform to e.g. safeguard and issue early warnings to firemen. Since sensors might be attached to UAVs, vehicles and personnel, the AWARE GSN is characterized by a combination of static and mobile nodes. Together with the high level of QoS that is required for safeguard applications, this is a major challenge from GSN design perspective.

Another challenge is energy-efficiency of the wireless sensors and the networking protocols. Although in the majority of wireless sensor network literature energy-efficiency is considered important for network lifetime, our reason for energy-efficiency is more related to the autonomous deployment aspect of the GSN and the weight of nodes to be carried by UAVs: the better the energy-efficiency the lesser battery capacity is required. Energy-efficiency has thus an influence on the weight of sensor nodes and the efficiency of autonomous deployment. Next, we discuss required characteristics of the wireless sensor mesh networking and node collaboration for event detection and reporting via AWARE middleware publish/subscribe.

\section{B. Characteristics}

The ground sensor nodes take care of the fault tolerant mesh networking in the GSN to provide communication services between nodes. Adding more of these devices makes sensing and wireless communication in general more reliable. The scalable, self-organizing network adapts to changes in traffic, radio conditions, device status and location. These devices self-organize at power-up and quickly reconfigure as devices join, leave or move around in the network. They also adapt to changes in the network traffic and propagation conditions. These capabilities enable mobility of individual devices or the entire network, and minimize installation effort, which is one of the prominent requirements of the AWARE platform.

Several anomalies can occur in the wireless sensor network that can impair their communication functionality. The target field that is supposed to be $100 \%$ covered by the densely deployed nodes may have coverage holes, areas not covered by any node, due to random aerial deployment creating voids, presence of obstructions, and, more likely, node failures etc. Similarly, nodes may not be able to communicate correctly if routing holes, areas devoid of any nodes, exist in the deployed topology. Thus, the network fails to achieve its objectives if some of the nodes cannot sense or report the sensed data. GSN needs some indications for network connectivity to recover node failures and to report these holes to the self-deployment system in the AWARE platform.

\section{Layers}

The layers of the GSN communication protocol stack can be seen in Figure 3. To ensure multi-hop networking between the nodes, the wireless medium must be shared between multiple nodes. The data link layer provides a set of communication rules i.e. the medium access control (MAC) protocol, to establish conflict-free and interference-free communication between nodes, while limiting latency and energy-wastage. The data link layer controls the transceiver in such way that communication is reliable. Since it is closely involved in controlling one of the most energy-consuming components of the node hardware, its optimization has tremendous influence on the energy-efficiency of nodes. In AWARE, an energy-efficient and scalable MAC protocol that can cope with mobility of nodes have been designed.

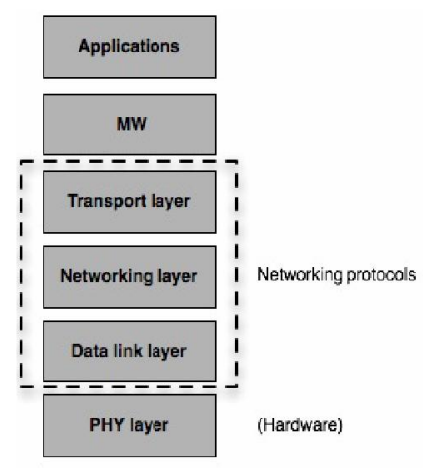

Fig. 3. Components in GSN protocol stack

The networking layer -often called routing protocol in wireless sensor network literature- takes care of efficient routing of data in the multi-hop network. Nodes between source and destination forward the data appropriately (and no 
higher layers are involved in this process). The transport layer ensures reliable data transfer between source and sink of the data. Node failures (due to harsh environmental conditions) and communication failures (due to mobility of nodes or interference or even malicious interference) make the topology of the AWARE GSN very dynamic. We assume redundancy in the network in order to be robust against these dynamics. In AWARE, these layers have to be designed to be able to handle dynamics and to be self-healing, when conflicts occur. Additionally, QoS aspects are taken into account.

\section{Event detection in the AWARE GSN.}

As discussed before, the GSN detects events (e.g. fire) by means of sensors and wirelessly communicates the event description. The wireless sensors collaborate to deliver the event to a GSN gateway, which communicates via the middleware publish/subscribe interface to other entities in the AWARE platform. The two basic operations, event detection and data dissemination, must be well studied. A localized and distributed detection algorithm is highly preferred for the AWARE system. The basic idea of distributed detection is to have a number of independent sensors each makes a local decision (typically a binary decision) and then to combine these decisions at a fusion sensor to generate global consensus and to set -based upon e.g. the importance of the event- the required QoS to be used by the networking protocols. Such an event-detection algorithm must also be fault tolerant. It is crucial to design and employ an energy-efficient data dissemination protocol.

\section{THE SELF-DEPLOYMENT SYSTEM.}

The self-deployment system is based on autonomous helicopters, which can transport loads (e.g. sensors) and deploy them at specified positions. Depending on the mass of the load the system can be operated in different modes, where one, two or three helicopters are involved in the transportation task. The load is attached to the helicopters by the means of flexible ropes. The advantages of the proposed self-deployment system composed of multiple helicopters are the good scalability and the moderate costs (compared to a larger UAV, which is able to carry the load alone).

The system is based on the MARVIN (Multi-purpose Aerial Robot Vehicle with Intelligent Navigation) UAVs, which are developed and used by the Technische Universität Berlin since 1997 and were upgraded to their current version (MarkIII) to meet the requirements of the AWARE project.

The MARVIN MarkIII (like the MarkII) is based on the CB5000 model. To archive autonomous flight capabilities the helicopters are equipped with a Novatel OEM4 GPS, a Microstrain GX-1 IMU and a $400 \mathrm{Mhz}$ PC104 real-time computer. All equipment is mounted to the helicopters using an aluminum frame made of strut profiles, which allows the quick mounting of additional hardware (e.g. fire sensors, cameras) or the quick replacement of defective hardware.

Windows CE 5.0 is used as real-time operation system and all software is embedded into a software framework called
Real-Time-Base-System (RTBS). The RTBS allows the integration of Matlab/Simulink generated code and handwritten C-code using extended black board architecture for data exchange and event triggering.

The controller for the uncoupled helicopters was developed using the generalized, model based control approach described in [7] and is applicable for the control of vertical take off and landing UAVs, like quad-rotors or helicopters.

Figure 4 shows the node deployment device, mounted to the helicopter. The design was inspired by the deployment device used in [8]. The functionality of the device is the same as for candy bar automats: A short wire is attached to the node ending in a metal grommet. This grommet is attached to the right end of the steel spring and the clockwise rotation of the spring moves the grommet (and the node) further onto the spring. This procedure allows attaching several nodes to the helicopter. During the dropping maneuver the spring rotates counterclockwise until the rightmost grommet is moved beyond the end of the spring and the node is released.

The node deployment device was already successfully used during the experiments in Utrera 2007 (see Fig. 8 where the dropping of a node is shown). The controller for multiple coupled helicopters is still under development.

\section{THE AWARE FUNCTIONALITIES.}

The main functionalities of the AWARE platform are co-operative surveillance and tracking. The goal of co-operative surveillance is to create a general probabilistic framework for the detection of events using static and mobile sensors. The following sensors are used in the AWARE platform: visual and infrared images from cameras mounted on UAVs, GCNs and sensors of scalar magnitude such as temperature, humidity, COx and NOx.

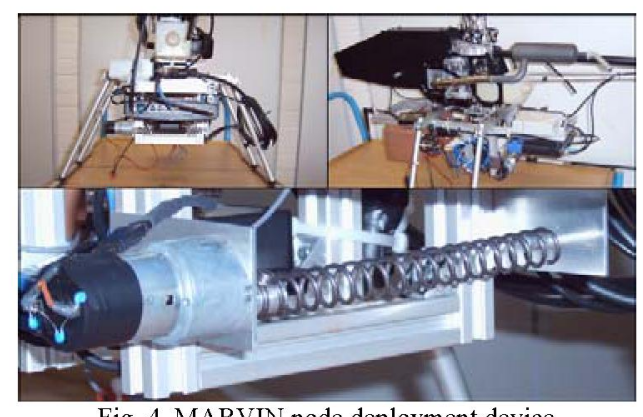

Fig. 4. MARVIN node deployment device

AWARE integrates the visual and infrared detection tools developed in previous projects [2] and has developed new tools. One of these tools is the smoke detection based on the integrated analysis of color, motion and texture (see [9]).

The goal of the co-operative tracking is to create a general probabilistic framework for tracking events manually or automatically detected by using the sensors provided by the AWARE platform. The estimation of the position and orientation of the UAVs, and mobile objects in general, is a 
key issue in surveillance and tracking. In addition of the GPS and navigation sensors of UAVs, the node signal strength from the WSN nodes is used for the localization of the nodes. A tracking tool is being developed. This tool includes a visual tracker based on the following steps: color segmentation, blob segmentation, detection based on the shape, size and color of the blobs, and position estimation. An Extended Kalman Filter (EKF) has been implemented for the position tracking [9].

Furthermore, a new technique for the estimation of the position of the static nodes of the WSN has been developed. This technique is based on having on-board the mobile robot one sensor node and "project" the position of the node, which can be known by means of the GPS of the robot, to the WSN through the signal strength. The node position estimation has been implemented by using a particle filter. The implemented algorithm showed good convergence to the correct solution, coherent standard deviation and small number of particles. New methods for the position estimation and tracking of mobile nodes using faster filtering techniques are being researched.

A decentralized architecture for co-operative perception is being developed. The perception software for each AWARE entity (GCNs, UAVS and WSN) process locally the sensor data in order to reduce the amount of data transferred through the network. All the instances share the perception information extracted from their local sensors. Each instance is able to improve its knowledge of the environment. All the events are represented by their estimated position in mean and standard deviation. The framework can be used with different believe representations (Grids, Particle Filters, Extended Kalman Filters). An Extended Kalman Filter (EKF) is currently being implemented in each perception software instance to fuse their own estimation of the event and the information gathered from the rest of systems. The EKF prediction is used by the perception software instances to improve the detection/tracking.

\section{EXPERIMENTS.}

The first general AWARE experiments took place in Utrera (near Seville, Spain) in March 2007. The main objectives were to obtain feedback for the design of the AWARE platform and to record data to develop the different subsystems and functionalities. Figure 6 shows the layout and a photograph of the scenario.

A three-floor building was simulated by means of the structure shown in Figure 5. There is a ladder providing access of the firefighters to the three levels. A closed room was also installed in the first level. Fire-fighters, and fire trucks participated in the experiments. Smoke and fire machines were used to simulate the fires. The experiments are described in the following.

\section{A. Fire detection, confirmation and localisation}

The first step is the detection of a fire inside the building by means of the wireless sensor network deployed in the structure. A node of the wireless sensor network detected the fire and generated an alarm. The Marvin autonomous helicopter was sent to confirm the alarm. The general characteristics of this autonomous system were presented in section V. Marvin took-off and flew to the building to confirm the alarm, by using an on-board infrared camera. At the same time the ground camera nodes detected the smoke. The smoke detection technique is presented in [9]. Then, the fire was confirmed. Moreover, computer vision was used to compute the localization of the fire from the previous calibration of the static GCN and the knowledge of the position and orientation of the autonomous helicopter that were transmitted through the middleware. The relative orientation of the infrared camera was known with respect to the helicopter frame (no pan and tilt device was used in the Marvin helicopter). Then, automatic geo-referencing of the objects in the images of the infrared cameras can be applied.
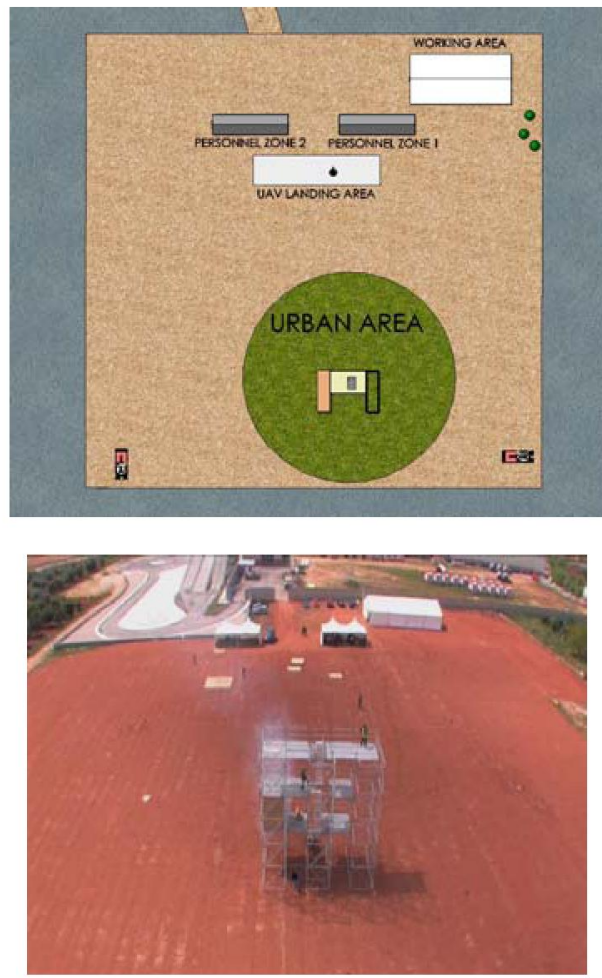

Fig.5 . 2007 AWARE experimentation scenario

\section{B. Monitoring}

Once the alarm was confirmed the Flying-Cam helicopter flew to monitor the fire and the global scenario (see Fig. 6). Its main features are: $1.8 \mathrm{~m}$ main rotor diameter, $15 \mathrm{Kg}$ weight (ready to fly), methanolengine, gyro-stabilized head containing the camera of choice. The helicopter is equipped with an advanced autopilot [10] that allows the helicopter to fly following velocity commands given by the operator. The fire monitoring phase includes the evaluation of the fire by using the images transmitted by the helicopter and the tracking of the people and vehicles around the fire. This perception functionality is presented in [9]. 
Figure 6 also illustrates the sensor data obtained in the experiment. The green line in the temperature diagram corresponds to the node near the fire.

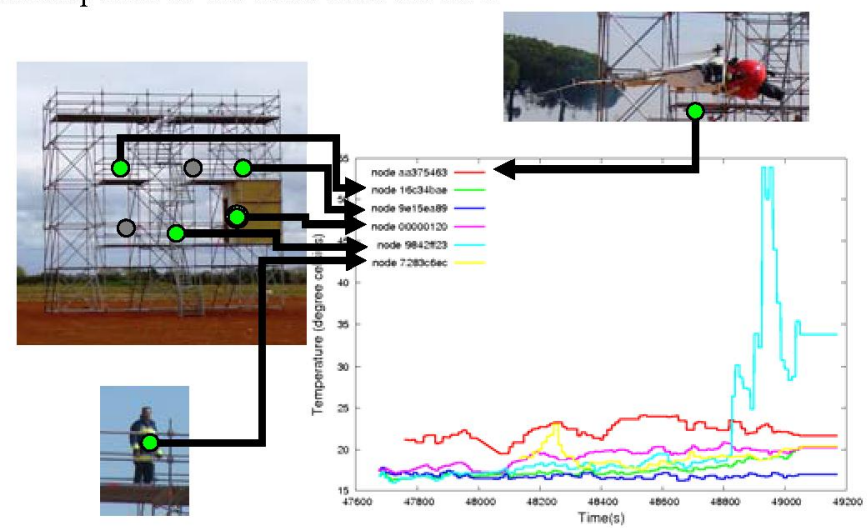

Figure 6: WSN data (temperature) from the nodes in the structure, the node carried by the firemen and the node on-board the Flying-Cam helicopter.

\section{Node deployment}

Preliminary experiments have been conducted by deploying wireless sensor nodes with the Marvin autonomous helicopter (see V). Several deploying experiments have been conducted. Figure shows Marvin deploying one of the sensor nodes of the WSN in section III. It was noticed that the nodes were wirelessly connected when flying on-board the helicopter and where they were in the air. However, communications problem appear after dropped. This was possibly due to the position of the antenna on the ground. Then, new enclosures should be applied to guarantee the verticality of the antenna on-ground. Moreover, new antenna technologies could be applied in the future.

\section{CONCLUSIONS}

The integration of Unmanned Aerial Vehicles, Ground Camera Nodes and WSNs with static and mobile nodes offers many potentialities for Disaster Management Scenarios, including Search and Rescue Operations. This paper has presented a system for the integration of these heterogeneous components and some of its functionalities for the operation in these environments. The paper provides an overview of the elements of this system and summarizes some results obtained in the first general experiments. The presentation will include videos and results of these experiments as well as the lessons learnt for the final implementation that will include the full self-deployment and connectivity repairing of the WSN by means of autonomous helicopters.

\section{ACKNOWLEDGEMENT}

AWARE is funded by the European Commission under contract IST 2006-33579. The authors thank the contribution of the Seville Fire Fighting Service for the Experiments described in Section VI.

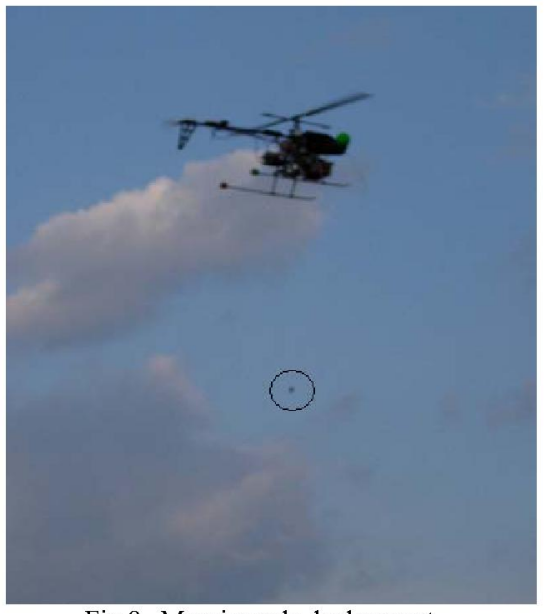

Fig.8 . Marvin node deployment

\section{REFERENCES}

[1] Ollero, A.and L. Merino. Control and perception techniques for aerial robotics, Annual Reviews in Control, Vol. 28, Issue 2, Pages $167-178,2004$.

[2] Ollero A., S. Lacroix, L. Merino, J. Gancet, J. Wiklund, V. Remuss, I. Veiga, L.G. Gutierrez, D.X. Viegas, M.A. Gonzalez, A. Mallet, R. Alami, R. Chatila, G. Hommel, F.J. Colmenero, B. Arrue, J. Ferruz, R. Martinez de Dios, and F. Caballero. "Architecture and perception issues in the COMETS multi-UAV project". IEEE Robotics and Automation Magazine, Vol 12, No. 2, pp 46-57, June 2005

[3] Glaser S. D., "Some real-world applications of wireless sensor nodes Proceedings of SPIE Symposium on Smart Structures \& Materials/ NDE 2004", San Diego, California, March 14-18, 2004.

[4] Havinga P., J. C. Hou, M. Srivastava, and F. Zhao "Wireless Sensor Networks", Guest editorial IEEE Wireless Communication Magazine, December 2004

[5] Li Q. and D. Russ, "Sending messages to mobile users in disconnected ad-hoc wireless networks." in Proceedings of 6th ACM Annual International Conference on Mobile Computing and Networking, MobiCom 2000, Boston, MA, pp. 44-55, August $6-112000$.

[6] Marrón P.J., Andreas Lachenmann, Daniel Minder, Jörg Hähner, Robert Sauter and Kurt Rothermel. "TinyCubus: A Flexible and Adaptable Framework for Sensor Networks". Proceedings of the $2^{\text {nd }}$ European Workshop on Wireless Sensor Networks. 2005.

[7] Kondak K., Bernard M., Meyer N., "Autonomous Flying VTOL-Robots: Modeling and Control" in Proceedings of IEEE International Conference on Robotics and Automation 2007.

[8] Corke, P. Hrabar, S. Peterson, R. Rus, D. Saripalli, S. Sukhatme, G. "Autonomous deployment and repair of a sensor network using an unmanned aerial vehicle" in Proceedings. ICRA IEEE International Conference on Robotics and Automation 2004.

[9] Capitán J. Mantecón D., Soriano P. and A. Ollero. Autonomous perception techniques for urban and industrial fire scenarios. To appear in the Proceedings of the IEEE Search and Rescue Robotics Conference, Rome, 2007.

[10] La Civita M., G. Papageorgiou, W. C. Messner, T. Kanade Integrated Modeling and Robust Control for Full-Envelope Flight of Robotic Helicopters. Proceedings of the 2003 IEEE International Conference on Robotics and Automation, vol.1, pp 552- 557, Taipei, Taiwan, 14-19 Sept. 2003 